Published in final edited form as:

Invest Ophthalmol Vis Sci. 2009 December ; 50(12): 5872-5879. doi:10.1167/iovs.09-3675.

\title{
In vivo imaging of microscopic structures in the rat retina
}

\author{
Ying Geng ${ }^{1 a, b}$, Kenneth P. Greenberg ${ }^{2}$, Robert Wolfe ${ }^{1 a}$, Daniel C. Gray ${ }^{3}$, Jennifer J. \\ Hunter $^{1 a}$, Alfredo Dubra ${ }^{1 a}$, John G. Flannery ${ }^{2}$, David R. Williams ${ }^{1 a}$, and Jason Porter ${ }^{4}$ \\ ${ }^{1 a}$ Center for Visual Science, University of Rochester, Rochester, NY \\ ${ }^{1 b}$ The Institute of Optics, University of Rochester, Rochester, NY \\ ${ }^{2}$ Department of Molecular \& Cell Biology, UC Berkeley, Berkeley, CA \\ ${ }^{3}$ Optos plc, Scotland KY11 8GR, United Kingdom \\ ${ }^{4}$ College of Optometry, University of Houston, Houston, TX
}

\section{Abstract}

Purpose-The ability to resolve single retinal cells in rodents in vivo has applications in rodent models of the visual system and retinal disease. We have characterized the performance of a fluorescence adaptive optics scanning laser ophthalmoscope (fAOSLO) that provides cellular and subcellular imaging of rat retina in vivo.

Methods-Green fluorescent protein (eGFP) was expressed in retinal ganglion cells of normal Sprague Dawley rats via intravitreal injections of adeno-associated viral vectors. Simultaneous reflectance and fluorescence retinal images were acquired using the fAOSLO. fAOSLO resolution was characterized by comparing in vivo images with subsequent imaging of retinal sections from the same eyes using confocal microscopy.

Results-Retinal capillaries and eGFP-labeled ganglion cell bodies, dendrites, and axons were clearly resolved in vivo with adaptive optics (AO). AO correction reduced the total root mean square wavefront error, on average, from $0.30 \mu \mathrm{m}$ to $0.05 \mu \mathrm{m}$ (1.7-mm pupil). The full width at half maximum (FWHM) of the average in vivo line-spread function (LSF) was $\sim 1.84 \mu \mathrm{m}$, approximately $82 \%$ greater than the FWHM of the diffraction-limited LSF.

Conclusions-With perfect aberration compensation, the in vivo resolution in the rat eye could be $\sim 2 \times$ greater than that in the human eye due to its large numerical aperture $(\sim 0.43)$. While the fAOSLO corrects a substantial fraction of the rat eye's aberrations, direct measurements of retinal image quality reveal some blur beyond that expected from diffraction. Nonetheless, subcellular features can be resolved, offering promise for using $\mathrm{AO}$ to investigate the rodent eye in vivo with high resolution.

\section{Introduction}

Naturally occurring, transgenic and knockout rodent models are instrumental in the study of retinal disease mechanisms and in the development of treatments for human retinal dystrophies. To date, the majority of studies using rodent disease models rely upon retinal histopathology to follow disease progression and the effect of candidate therapies. Histopathology yields high resolution images and morphometric estimates of surviving retinal cells; ${ }^{1}$ however, the major drawback of this approach is that it does not allow longitudinal studies in the same animals.

In vivo imaging of the rodent retina offers the possibility to visualize disease processes and progression in individual animals and to reduce the effects of animal to animal variation, background lighting and genetic background. For example, it has been shown that in various neurodegenerative diseases, substantial modifications in the morphology of axons and dendrites can take place well before cell death. $2,3,4,5,6,7,8$ These observations highlight the 
need to develop high resolution in vivo imaging techniques capable of resolving subcellular structures (such as individual axons and dendrites) in rodent eyes.

The resolution of in vivo imaging is limited by the optical quality of the rodent eye. Compared with the human eye, rodent eyes have smaller axial lengths, higher optical powers, larger average refractive errors and larger numerical apertures (NA) [see Table 1]. Rats typically possess a large hyperopic refractive error. Retinoscopy measurements have shown refractive errors in the range of $+5 \mathrm{D}$ to $+15 \mathrm{D}$ in albino rats, with a strong dependence on the strain (Irving EL, et al. IOVS 2005;45:ARVO E-Abstract 4334). A dilated rodent eye also has a larger numerical aperture than a dilated human eye. Thus, in theory, one could resolve smaller retinal features with a perfect correction of the eye's aberrations in a dilated rodent eye than in a dilated human eye.

Many studies have used fluorescence microscopy, fundus photography, two photon microscopy, confocal microscopy, or scanning laser ophthalmoscopy (SLO) to image the living rodent retina, allowing the visualization of structures such as blood vessels, capillaries, nerve fiber bundles, photoreceptors, retinal ganglion cells (RGCs), retinal pigment epithelial (RPE) cells and microglial cells. ${ }^{21}, 22,23,24,25,26,27,28,29,30,31,32,33,34,35,36,37,38,39$ Fluorescently labeled RGCs have been imaged in the rodent retina in vivo over a wide field, $21-28,36,38,39$ with some studies showing the apparent loss of ganglion cells in diseased retina. $23,24,26,27,38,39$ One report recently used a confocal laser scanning microscope to image ganglion cells and processes in vivo in transgenic mice that expressed yellow fluorescent protein (YFP) in a small subset of RGCs. ${ }^{33}$ Resolution in all of these studies could be improved by correcting the eye's aberrations with adaptive optics (AO) so that many fine features that could previously be resolved only in excised retina could now be imaged in vivo. Adaptive optics ophthalmoscopes have enabled near diffraction-limited imaging of cellular structures (such as individual photoreceptors, ganglion cells, and RPE cells) in living human and nonhuman primates, ${ }^{40,41,42,43,44}$ as well as the resolution of subcellular features (such as ganglion cell axons and dendrites) in living non-human primates. ${ }^{42,43}$ Recently, Biss et al. fluorescently imaged mouse capillaries and microglia cells using an adaptive optics biomicroscope, showing that some of the benefits of adaptive optics found in primates can be realized in rodent eyes. ${ }^{31}$

We describe here a fluorescence scanning laser ophthalmoscope equipped with adaptive optics (fAOSLO) for the rat eye. We show that cellular and subcellular features in the rat retina, such as fine capillaries and individual fluorescently-labeled ganglion cell dendrites and axons, can be imaged. The in vivo resolution of our instrument was subsequently determined via confocal images of flatmounts of the enucleated retinas.

\section{Methods}

Normal Sprague-Dawley rats from 2 to 21 months of age were used in this study. All animals were handled according to the Association for Research in Vision and Ophthalmology Statement for the Use of Animals in Ophthalmic and Vision Research and to the guidelines of the University Committee on Animal Resources at the University of Rochester.

\section{eGFP labeling of RGCs}

3 to $5 \mu \mathrm{L}\left(0.5-5 \times 10^{13} \mathrm{vg} / \mathrm{ml}\right)$ intravitreal injections of adeno-associated serotype 2 (AAV2) viral vectors containing a human synapsin promoter were used to express enhanced green fluorescent protein (eGFP) in ganglion cells of normal adult Sprague-Dawley rats. Vectors were produced and purified as described by Zolotukhin et al. ${ }^{45}$ Rats were anesthetized using a ketamine $(\sim 65 \mathrm{mg} / \mathrm{kg})$ and xylazine $(\sim 8 \mathrm{mg} / \mathrm{kg})$ cocktail and intravitreal injections were made through the sclera using a Hamilton syringe fitted with a $1 \mathrm{~cm}, 32$-gauge needle (described in 
detail by Greenberg et al. ${ }^{46}$ ). Retinal ganglion cells displayed visible in vivo eGFP fluorescence approximately 4 weeks post-injection which persisted for the life of the animal. Fluorescence fundus photographs were acquired using a modified fundus camera ${ }^{47}$ or a RetCam (Clarity Medical Systems, Inc., Pleasanton, CA, USA) to image ganglion cell eGFP labeling prior to high-resolution fAOSLO imaging. We estimate that over $90 \%$ of the retinal ganglion cells were transduced by the AAV2 viral vectors as the entire retina is contacted by the AAV vectors following an intravitreal injection.

\section{Preparation for in vivo AOSLO imaging}

Rats were anesthetized with isofluorane gas (2-3\%) and placed on a heating pad prior to in vivo imaging. Pupils were dilated with one drop of $2.5 \%$ phenylephrine (Neo-Synephrine) and one drop of $0.5 \%$ tropicamide. A 0 to +20 D rigid contact lens (Unicon Corporation, Osaka, Japan) was placed on the eye to maintain corneal hydration and reduce refractive error during in vivo fAOSLO imaging. Slit lamp imaging and retinoscopy were used to determine the base curve and refractive power of the contact lens required for each eye.

Rats were stabilized for imaging on a custom stereotaxic apparatus with earbars. A three-axis translation stage was used to align the rat's pupil with the exit pupil of the fAOSLO. A goniometer and a rotation mount (with their centers of rotation centered in space on the eye's pupil) were used to rotate the angle of the entrance beam to image different retinal locations. Large-scale features (such as blood vessels) observed in the previously acquired fluorescent fundus photos (see Fig. 3) were used as landmarks to navigate to desired retinal locations and to track the same location over multiple imaging sessions.

\section{The rat confocal fAOSLO}

High-resolution retinal images were obtained using a modified fAOSLO initially developed for imaging human and non-human primates. ${ }^{42}$ The scanning instrument (Fig. 1) incorporated an AO system consisting of a Shack-Hartman wavefront sensor and a 4- $\mu \mathrm{m}$ stroke MEMS deformable mirror (Boston Micromachines Corporation, Boston, MA, USA). A removable fold mirror (RFM) was inserted into the system and used to divert the imaging beam to a separate rodent arm with a pair of mirrors (M11, FM) that demagnified the pupil size to 3.5 $\mathrm{mm}$, or to an achromatic triplet lens (not shown) that demagnified the pupil size to $2.1 \mathrm{~mm}$. The maximum imaging field sizes for the 3.5 and 2.1 - $\mathrm{mm}$ pupil sizes were 5.5 and 9 degrees, respectively.

A 794-nm superluminescent diode (SLD) [Superlum, Cork, Ireland] was used for reflectance imaging and the 488-nm line from an $\mathrm{Ar} / \mathrm{Kr}$ laser (Melles Griot, Carlsbad, CA, USA) was used for fluorescence excitation. Wavefront sensing of the rat eye's aberrations was performed using a 904-nm laser diode (Thorlabs Inc., Newton, NJ, USA). The maximum permissible exposure (MPE) for using these three sources simultaneously in the rat eye was calculated assuming that the rat retina possessed the same sensitivity as the human retina. These calculations also included a scaling factor that incorporated the differences in the spot size and retinal illuminance at the rat retina due to its higher numerical aperture (when compared to the human eye). Less then $80 \mu \mathrm{W}, 350 \mu \mathrm{W}$, and $20 \mu \mathrm{W}$ were used for the 904-nm laser diode, 794-nm SLD, and 488-nm laser, respectively. In aggregate, these combined powers were less than the MPE for a 60-minute exposure time and 3-degree field size according to the ANSI guide for the safe use of lasers. ${ }^{48,49}$

For in vivo imaging sessions, wavefront sensing and adaptive optics correction were performed over a 1.7-3.5 mm pupil size (depending on the magnification) at a rate of $15 \mathrm{~Hz}$. Residual spherocylindrical errors not corrected by the contact lens were corrected by placing trial lenses in front of the rat eye or in a conjugate system pupil plane (TL). To image different retinal 
layers, the imaging beam was defocused from the wavefront sensing plane using spherical trial lenses or by changing the focus of the deformable mirror. This defocus also incorporated the chromatic aberration between the imaging and wavefront sensing wavelengths.

\section{Image Acquisition and Analyses}

Single in vivo fluorescence frames typically possessed very low signal to noise ratio. Therefore, several fluorescence frames were summed to obtain an image with high signal to noise. To account for eye movements between different frames, images were registered using the shifts calculated from simultaneously acquired reflectance images. ${ }^{42}$ A cold mirror (CM2) was used to separate the visible and infrared light for simultaneous fluorescence and reflectance imaging. An avalanche photodiode (APD) [Perkin Elmer, Vaudreuil, QC, Canada], was used for reflectance detection at $794 \mathrm{~nm}$ and a photomultiplier tube (PMT) [H7422-40, Hamamatsu, Shizuoka-ken, Japan] was used for fluorescence detection of eGFP. Removable confocal pinholes $(\mathrm{CP})$ were placed at retinal conjugate planes in front of both detectors. Typical pinhole sizes ranged from 4.1-6.2 Airy disk diameters for reflectance imaging, and 2.3-7.0 Airy disk diameters for fluorescence imaging. Images were acquired at a rate of $27 \mathrm{~Hz}$.

As described previously, the maximum achievable imaging field size was 9 degrees. Though the resolution of this image is reduced, likely because it exceeds the isoplanatic patch size in the rat eye, the larger field of views were helpful for generating quick montages to identify landmarks or image larger features. Larger montages of nearby retinal locations were created by registering multiple images together using a custom written algorithm or by manually registering images using Adobe Photoshop (Adobe Systems Inc., San Jose, CA, USA). To more easily identify capillaries within the reflectance images, we computed the standard deviation of each pixel in the stabilized, registered images (Burns SA, et al. IOVS 2008; 49: ARVO E-Abstract 4512) using ImageJ (NIH, Bethesda, MD, USA). Changes in reflectance due to blood flow through the capillaries will result in greater variability (or a higher standard deviation) in the reflectance from the capillaries compared to the more static surrounding tissue.

To estimate the physical size of structures in the in vivo retinal images using its angular dimensions, we assumed that our rat eyes possessed the same focal length and vitreous refractive index as Hughes' rat schematic eye. ${ }^{16}$ The retinal spatial to angular conversion was calculated to be $58.1 \mu \mathrm{m} / \mathrm{deg}$. fAOSLO images in this paper were collected over a 3 degree or 4.7 degree field of view, corresponding to a physical width of $174.3 \mu \mathrm{m}$ or $273.1 \mu \mathrm{m}$, respectively, on the retina.

\section{Characterization of the in vivo transverse resolution}

Rats were sacrificed after multiple in vivo imaging sessions. Retinas were removed and placed as wholemounts on slides with coverslips and covered in mounting medium (Vectashield; Vector Laboratories, Burlingame, CA, USA). Wholemount images were acquired using a confocal microscope (Zeiss LSM510; Carl Zeiss, Inc., Germany). Through-focus stacks were taken at the same locations as the in vivo images using a $10 \times(0.30 \mathrm{NA})$ and $40 \times(1.2 \mathrm{NA})$ microscope objective, and maximum intensity projection images were generated for each stack. The pinhole size in the confocal microscope was adjusted to match the in vivo imaging parameters. Ex vivo images acquired with the 1.2 NA objective were taken to represent the true size of the object.

The in vivo transverse resolution of the fAOSLO was determined by calculating the in vivo line spread function (LSF). First, average cross-sectional profiles were measured across the same individual dendrites in the in vivo and ex vivo images. Both in vivo and ex vivo profiles were normalized with regard to the background level. The in vivo LSF was calculated by deconvolving the ex vivo dendrite profile from the corresponding in vivo dendrite profile. The 
in vivo resolution of the fAOSLO was defined as the full width at half maximum (FWHM) of the in vivo LSF.

\section{Results}

\section{In vivo AO imaging of capillaries in reflectance}

Adaptive optics correction typically reduced the higher order root mean square (RMS) wavefront error (as measured by the Shack-Hartman wavefront sensor for the $904 \mathrm{~nm}$ wavefront sensing beacon) from $\sim 0.5 \mu \mathrm{m}$ to $\sim 0.1 \mu \mathrm{m}$ for a $3-\mathrm{mm}$ pupil, and from $\sim 0.3 \mu \mathrm{m}$ to $\sim 0.05 \mu \mathrm{m}$ for a 1.7-1.9 mm pupil in our system. (For comparison, the Maréchal criterion of $\lambda / 14$ at the wavefront sensing wavelength is $\sim 0.06 \mu \mathrm{m}$.) In vivo images of retinal capillaries (Fig. 2) were acquired in reflectance following adaptive optics correction over a 1.85-mm pupil. All reflectance images correspond to the registered sum of 200 individual frames. Retinal features were difficult to discriminate prior to $\mathrm{AO}$ correction. After correcting for the rat eye's aberrations, capillaries were seen faintly in reflectance images.

The areas of blood flow can be visualized more clearly by computing the standard deviation of each pixel throughout the series of registered frames. Fig. 2(c) shows the standard deviation image calculated from the registered sequence that produced the image in Fig. 2b. Areas with high variability in their reflectance appear as bright regions in the image, while areas with relatively constant reflectance appear dark. The capillary network clearly emerged using this technique because of changes in capillary reflectance over time due to the movement of cells inside the capillary walls.

\section{In vivo fAOSLO imaging of fluorescently-labeled ganglion cells, axons and dendrites}

Prior to high-resolution fluorescence imaging with adaptive optics, wide field fluorescence fundus photos were acquired (see methods) to verify eGFP expression had commenced (Fig. 3). The ganglion cell layer was then imaged with the fAOSLO (Fig. 4). Large ganglion cell bodies could be observed in vivo without aberration correction (Fig. 4a), consistent with earlier studies. ${ }^{21-28}$ Adaptive optics correction of the rat's aberrations enabled us to readily distinguish individual RGC bodies that were difficult to differentiate prior to AO correction (arrows in Fig. $4 \mathrm{a}$ and $4 \mathrm{~b}$ ). In addition, AO allowed the clear visualization of RGC axons and dendrites (Fig. 4b). Using retinal landmarks, we were able to return to an identified retinal location in the same rodent, permitting the longitudinal documentation of individual RGCs. Fig. 4c shows a retinal image acquired at precisely the same retinal location in an individual rat that was reimaged 3 months later. The confocal pinhole size used for obtaining this image (2.3 Airy disk sizes) was $1 / 3$ the size of the one used for obtaining the image in Fig. 4b; thus, fewer photons were collected for the image in Fig. 4c. While defocus may not have been optimized in this image, the same cell bodies and processes were still resolved.

\section{Direct comparison of in vivo images to ex vivo images}

We compared the structure of individual RGCs observed in fAOSLO images obtained in vivo with the RGC structure seen in histological preparations of enucleated retina from the same eyes with confocal microscopy. Fig. 5 directly compares in vivo and ex vivo images of an RGC with a large diameter cell body. This is likely a giant cell (or RGA1 cell according to Huxlin and Goodchild's classification) ${ }^{50}$ Fig. 5a shows the area inside of the white square overlaid on the low resolution fAOSLO montage from Fig. 3. The in vivo image is a maximum intensity projection image generated from 3 separate in vivo images taken at different depths (focus step is 1.1 diopters or $16.3 \mu \mathrm{m}$ ). Ex vivo images are maximum intensity projection images generated from a through focus stack in the ganglion cell layer. The in vivo $0.25 \mathrm{NA}$ image recovers almost all of the information in the ex vivo $0.3 \mathrm{NA}$ image. 


\section{Characterization of in vivo transverse resolution}

The in vivo imaging resolution was characterized by comparing the in vivo images with corresponding ex vivo histological images. The in vivo line spread function (LSF) was computed over the four locations indicated by the four gray rectangles in Fig. 5a and 5c. Fig. 6a shows the average profiles measured across the same dendrite imaged in vivo (open circles with dashed line) and ex vivo using a 1.2 NA microscope objective (filled circles with solid line) from the rightmost gray rectangle in Fig. 5a and 5c. The normalized in vivo LSF (Fig. 6b) was calculated by deconvolving the ex vivo dendrite profile from the corresponding in vivo dendrite profile. This calculation assumes that the LSF of the 1.2 NA microscope objective is small and that the ex vivo image reveals the true dimensions of the dendrite. (This assumption is reasonable because the diffraction-limited LSF of the microscope is approximately 0.21 microns, which is 5 times smaller than the LSF estimated for diffraction-limited in vivo imaging. Simulations show that when imaged by a 1.2 NA objective, dendrites with FWHMs from 1.3 to $1.9 \mu \mathrm{m}$ broaden by less than 5\%.) For this location, the FWHM of the in vivo LSF was $1.77 \mu \mathrm{m}$. The average FWHM of the in vivo LSFs calculated at all four locations was 1.84 $\pm 0.30 \mu \mathrm{m}$ (average \pm standard deviation), approximately $82 \%$ larger than the FWHM of the diffraction-limited LSF $(1.01 \mu \mathrm{m})$.

\section{Discussion}

\section{Challenges of in vivo imaging of the rodent eye using adaptive optics}

It is challenging to achieve diffraction-limited imaging for the rat eye due to its small size, high optical power, and large refractive error. Due to the high optical power of the rodent eye, a large defocus value is needed for sectioning the retina. The total optical power for the human eye is $\sim 60 \mathrm{D}$, while the total optical power is $\sim 300 \mathrm{D}$ for the rat eye, yet the rodent retina is comparable in average thickness to that of human (typically $170 \mu \mathrm{m}$ vs. $250 \mu \mathrm{m}$, respectively). 16,51 Whereas only $\sim 2 / 3$ diopter is required to shift the focal plane in the human from the anterior to the posterior retina, an $\sim 11.5$ diopter change in optical power is required in the rat. Considering the different pupil sizes inherent in different species, the amount of Zernike defocus (in $\mu \mathrm{m}$ ) needed to section the whole retina is less than $1 \mu \mathrm{m}$ for the human eye and 5 $\mu \mathrm{m}$ for the rat eye. The large refractive error of the rodent eye poses another challenge for in vivo cellular imaging. We used trial lenses and contact lenses to compensate for spherocylindrical errors, but utilizing a larger stroke deformable mirror for refractive correction would provide a faster solution. Another issue to be considered when imaging with multiple wavelengths is the increased amount of chromatic aberration in the rat eye compared to that of the human eye; the chromatic aberration between $475 \mathrm{~nm}$ and $650 \mathrm{~nm}$ in the rat eye is reported to be 5.8 diopters. ${ }^{52}$ Last, but not least, wavefront sensing using reflective infrared light (which returns primarily from the photoreceptors) can cause additional uncertainties for effective $\mathrm{AO}$ correction when imaging retinal layers away from the photoreceptor layer. For example, in the rat, the $\sim 11.5$ diopter separation between the ganglion cell and photoreceptor layers could require modifications of other aberrations, such as spherical aberration, for best correction. Measurements of any variation in the wave aberration with different focal planes throughout the retina have yet to be made.

Careful animal handling is also important to achieve successful images. For fAOSLO imaging of the rat retina, we have found that isoflurane provides a better, more stable plane of anesthesia than when using ketamine and xylazine. The morbidity rates after anesthesia are lower, fewer eye movements are observed in the high magnification images, and the duration of the anesthesia can be longer (over 3 hours vs. 30 minutes) when using isoflurane. A heating pad helps to keep the animal warm and prevent transient, temperature-related cataract formation for imaging sessions longer than an hour. Using contact lenses to keep the eye hydrated eliminates the need to manually blink the eye or constantly hydrate the eye with drops, and 
avoids distortions in the wavefront due to degradations in the tear film or non-uniformities in the distribution of the hydrating drops. A contact lens also helps to reduce the lower-order aberrations of the eye. We found that the spherical refractive power and corneal curvature of our Sprague-Dawley rat eyes changed dramatically with age. Their spherical refractive power decreased from 10-20 diopters to approximately 5 diopters as they matured. Therefore, it is important to carefully select and match the base curve, diameter and power of the contact lens to the eye being imaged. Poor fitting contact lenses may degrade the spots in the wavefront sensor images, resulting in poor AO corrections and image quality.

\section{In vivo imaging of rat capillaries in reflectance}

Several studies have imaged rodent capillaries using fluorescein angiography, ${ }^{29-31,34,35}$ and Paques et al. attempted to image mouse capillaries in reflectance with an SLO ${ }^{30}$ In the present study, we imaged rat capillaries in reflectance with the fAOSLO. However, it is difficult to clearly differentiate all capillaries. The reflectance images shown in Fig. 2(a) and 2(b) appear blotchy with bright and dark areas. The exact origin of this blotchy appearance is not yet well understood, but they are stable features of these retinal images and may be related to out-offocus light from other retinal layers.

The standard deviation image processing technique highlights areas with high variability in the reflectance image, such as regions with blood flow. The combination of fAOSLO imaging and standard deviation image processing enables visualization of small capillaries in reflectance with image quality comparable to that of fluorescein angiography. This method presents a useful noninvasive alternative to performing fluorescein angiography for imaging capillaries in rodent and human eyes.

\section{Imaging subcellular features of rat ganglion cells in vivo}

Fluorescence imaging has been the most successful method to provide contrast for transparent retinal cells, such as ganglion cells in living eyes. Sabel et al. and Rousseau et al. used a modified confocal laser scanning microscope to first visualize rat RGCs that were retrogradely labeled via injections of fluorescent latex beads into superior colliculus. ${ }^{21,22}$ Naskar et al. and Thanos et al. monitored the death of retrogradely labeled rat RGCs using conventional fluorescence microscopy. ${ }^{23,24}$ Paques $e t$ al. visualized rat RGCs using fundus photography following retrograde transport of indocyanine green that was injected into the LGN. ${ }^{25}$ More recently, many in vivo studies have imaged RGCs using the SLO. Cordeiro et al. labeled rat and primate RGCs with intravitreal injections of annexin 5 and visualized RGC apoptosis using a confocal SLO ${ }^{26}$ Higashide et al. imaged and counted retrogradely labeled rat RGCs using a SLO. ${ }^{27}$ Recent reports from Leung et al. also used a confocal SLO to image mice RGCs that were intrinsically labeled in a strain of transgenic mice. ${ }^{28,39} \mathrm{All}$ of the above studies were able to visualize ganglion cell bodies. In another recent report, Walsh and Quigley were able to visualize ganglion cell bodies as well as their processes in a transgenic mouse that expressed YFP in a small subset of RGCs. ${ }^{33}$ Even though ganglion cell processes have been visualized in the mouse eye by selectively labeling a small subset of RGCs, ${ }^{33}$ the increased resolution afforded by adaptive optics enables us to resolve ganglion cell axons and dendrites in a densely labeled retina.

The major difference between the present and aforementioned studies is the use of $\mathrm{AO}$ to increase lateral resolution and provide higher magnification images. This method will potentially enable more accurate cell counting as cells that are difficult to differentiate in dense clumps in low resolution images can be individually distinguished using adaptive optics imaging. Adaptive optics imaging can also increase the amount of light detected through the confocal pinhole, translating into an increased axial resolution and signal to noise ratio (since a smaller pinhole size can be used in confocal imaging). The fAOSLO may also be used to 
track structures longitudinally, such as photoreceptors, capillaries and fine features of other neurons, in the rodent retina in vivo.

Of course, many in vivo rodent retinal imaging experiments can be done successfully without using AO. For example, a conventional fluorescence SLO is sufficient to image larger structures, such as blood vessels, ${ }^{29,30,34}$ or to monitor general patterns of ganglion cell and axon bundle loss in the eye. ${ }^{26,27,39}$ To image ganglion cell dendrites in a sparsely labeled retina, one can use a confocal microscope. ${ }^{33}$ To image ganglion cell dendrites, perform exact cell counts in a densely labeled retina, or resolve other subcellular features, AO and confocal imaging are very helpful. Adaptive optics provides rapid, automated correction of all the eye's monochromatic aberration, including both lower and higher order aberrations, that are difficult to correct for using other methods.

\section{In vivo transverse resolution}

To our knowledge, the present study is the first to characterize the in vivo imaging resolution in rodent eyes. Despite the fact that the residual RMS wavefront error was $\sim 0.05 \mu \mathrm{m}$ after AO correction, the in vivo transverse LSF was found to approximately $82 \%$ larger than expected. There are a number of factors that may account for this difference. Our leading candidate is non-optimized imaging defocus. As mentioned before, at the location shown in Fig.5, three separate in vivo images were taken at focus steps of $16.3 \mu \mathrm{m}$. In comparison, the depth of focus is calculated to be $10.4 \mu \mathrm{m}$. Simulations show that a defocus value of $\pm(16.3 / 2) \mu \mathrm{m}$ along the axial direction is consistent with the $82 \%$ error. Therefore finer focus steps would be needed to achieve better resolution. Another possible factor is that the wavefront sensor may not be capturing all sources of image blur due to the fact that wavefront sensing and imaging are done in two different retinal layers (as previously discussed). Other possible contributing factors include accounting for eye movements within an individual frame, additional complications in wavefront sensing due to non common-path errors and scattering. Thus, although we have achieved an in vivo resolution in which we can resolve subcellular features such as axons and dendrites, there is promise for further resolution improvement.

In this report, the in vivo imaging resolution was characterized over a $1.7-\mathrm{mm}$ pupil size $(0.25$ $\mathrm{NA}$ ). We have also imaged ganglion cells and processes using a 3-mm pupil (0.43 NA) with residual RMS wavefront errors on the order of $0.1 \mu \mathrm{m}$ after AO correction. Although we were unable to characterize the in vivo resolution using images acquired through this 3-mm pupil size (due to insufficient labeling and contrast in these retinal images), the in vivo resolution could be higher with a 3-mm pupil (as opposed to a 1.7- $\mathrm{mm}$ pupil) due to the larger diffraction limit. It may eventually be possible to exceed the diffraction limit imposed by the dilated pupil of the eye. Methods to achieve this improvement in retinal imaging are under development. ${ }^{53}$

\section{Future applications}

The ability to resolve subcellular features such as individual ganglion cell axons and dendrites in rodents in vivo provides us the opportunity to detect early changes in the retina on a microscopic level in disease models and to investigate fundamental questions about retinal disease pathophysiology. For example, adaptive optics imaging may help to determine if remodeling occurs during glaucoma, whether axonal degeneration precedes dendrite degeneration, what the pattern of cell death is and whether vascular degeneration proceeds RGC death. While we have concentrated on ganglion cell imaging in this study, this level of resolution indicates that it may be possible to use the fAOSLO to image individual Müller glial cells, RPE cells, and rod and cone photoreceptors that are fluorescently labeled in transgenic rodents, ${ }^{54,55}$ or using cell-specific adeno-associated virus (AAV) and lentiviral vectors. ${ }^{46,}, 56$, 57, 58 
The mouse is the most widely used animal model for human retinal degenerations. A similar imaging method might also be applied to image multiple cells types in normal and transgenic mice. With the current powerful molecular techniques that exist for engineering the mouse, fAOSLO imaging of mouse models has potential for investigating disease mechanisms and evaluating the efficacy of drug therapies. Finally, it may also be possible to combine fAOSLO imaging with optical methods used to image the functional activity of cells, allowing optical recordings of the activity of single or ensembles of retinal cells in vivo.

\section{Acknowledgments}

The authors thank Benjamin Masella, William H. Merigan, Andrew Winterborn, Wendy Bates, Wanli Chi, Mina Chung, Richard T. Libby, Stephen Burns, Joseph Stamm, Terry Schaefer, and Lana Nagy for assistance. This research was supported financially by the National Institute for Health, Bethesda, Maryland (NIH EY014375), Foundation Fighting Blindness, the Texas Advanced Research Program (G096152), and the National Science Foundation Science and Technology Center for Adaptive Optics (Santa Cruz, California), managed by the University of California at Santa Cruz (cooperative agreement no.: AST-9876783).

\section{References}

1. LaVail MM, Battelle BA. Influence of eye pigmentation and light deprivation on inherited retinal dystrophy in the rat. Exp Eye Res 1975;21:167-192. [PubMed: 1164921]

2. Whitmore AV, Libby RT, John SWM. Glaucoma: Thinking in new ways-a rôle for autonomous axonal self-destruction and other compartmentalised processes? Prog Retin Eye Res 2005;24:639662. [PubMed: 15953750]

3. Howell GR, Libby RT, Jakobs TC, et al. Axons of retinal ganglion cells are insulted in the optic nerve early in DBA/2J glaucoma. J Cell Biol 2007;179:1523-1537. [PubMed: 18158332]

4. Nickells RW. From ocular hypertension to ganglion cell death: a theoretical sequence of events leading to glaucoma. Can J Ophthalmol 2007;42:278-87. [PubMed: 17392853]

5. Buckingham BP, Inman DM, Lambert W, et al. Progressive ganglion cell degeneration precedes neuronal loss in a mouse model of glaucoma. J Neurosci 2008;28:2735-2744. [PubMed: 18337403]

6. Weber AJ, Kaufman PL, Hubbard WC. Morphology of single ganglion cells in the glaucomatous primate retina. Invest Ophthalmol Vis Sci 1998;39:2304-2320. [PubMed: 9804139]

7. Morgan JE, Uchida H, Caprioli J. Retinal ganglion cell death in experimental glaucoma. $\mathrm{Br}$ J Ophthalmol 2000;84:303-310. [PubMed: 10684843]

8. Jakobs TC, Libby RT, Ben Y, John SWM, Masland RH. Retinal ganglion cell degeneration is topological but not cell type specific in DBA/2J mice. J Cell Biol 2005;171:313-325. [PubMed: 16247030]

9. Oliveira C, Harizman N, Girkin CA, et al. Axial length and optic disc size in normal eyes. Br J Ophthalmol 2007;91:37-39. [PubMed: 16987902]

10. Stenstrom S. Woolf D. Investigation of the variation and correlation of the optical elements of humans eyes. Am J Optom Arch Am Acad Optom 1948;48:344-345.

11. Sorsby A, Sheridan M, Leary GA, Benjamin B. Vision, visual acuity, and ocular refraction of young men. Br Med J 1960;1:1394-1398. [PubMed: 13832932]

12. Jackson E. Norms of refraction. J A M A 1932;98:132-137.

13. Sperduto RD, Seigel D, Roberts J, Rowland M. Prevalence of myopia in the United States. Arch Ophthalmol 1983;101:405-407. [PubMed: 6830491]

14. Wang Q, Klein BE, Klein R, Moss SE. Refractive status in the Beaver Dam Eye Study. Invest Ophthalmol Vis Sci 1994;35:4344-4347. [PubMed: 8002254]

15. Slataper FJ. Age norms of refraction and vision. Arch Ophthalmol 1950;43:466-481.

16. Hughes AA. schematic eye for the rat. Vision Res 1979;19:569-588. [PubMed: 483586]

17. Schmucker C, Schaeffel F. A paraxial schematic eye model for the growing C57BL/6 mouse. Vision Res 2004b;44:1857-1867. [PubMed: 15145680]

18. Remtulla S, Hallett PE. A schematic eye for the mouse, and comparisons with the rat. Vision Res 1985;25:21-31. [PubMed: 3984214] 
19. Garcia de la Cera E, Rodriguez G, Llorente L, Schaeffel F, Marcos S. Optical aberrations in the mouse eye. Vision Res 2006;46:2546-2553. [PubMed: 16516259]

20. Tejedor J, de la Villa P. Refractive changes induced by form deprivation the mouse eye. Invest Ophthalmol Vis Sci 2003;44:32-36. [PubMed: 12506052]

21. Sabel BA, Engelmann R, Humphrey MF. In vivo confocal neuroimaging (ICON) of CNS neurons. Nat Med 1997;3:244-247. [PubMed: 9018248]

22. Rousseau V, Engelmann R, Sabel BA. Restoration of vision III: soma swelling dynamics predicts neuronal death or survival after optic nerve crush in vivo. Neuroreport 1999;10:3387-3391. [PubMed: 10599850]

23. Naskar R, Wissing M, Thanos S. Detection of early neuron degeneration and accompanying microglial responses in the retina of a rat model of glaucoma. Invest Ophthalmol Vis Sci 2002;43(9):29622968. [PubMed: 12202516]

24. Thanos S, Indorf L, Naskar R. In vivo FM: using conventional fluorescence microscopy to monitor retinal neuronal death in vivo. Trends Neurosci 2002;25:441-444. [PubMed: 12183199]

25. Paques M, Genevois O, Régnier A, et al. Axon-tracing properties of indocyanine green. Arch Ophthalmol 2003;121:367-370. [PubMed: 12617707]

26. Cordeiro MF, Guo L, Luong V, et al. Real-time imaging of single nerve cell apoptosis in retinal neurodegeneration. Proc Natl Acad Sci U S A 2004;101:13352-13356. [PubMed: 15340151]

27. Higashide T, Kawaguchi I, Ohkubo S, Takeda H, Sugiyama K. In vivo imaging and counting of rat retinal ganglion cells using a scanning laser ophthalmoscope. Invest Ophthalmol Vis Sci 2006;47:2943-2950. [PubMed: 16799037]

28. Leung CK, Lindsey JD, Crowston JG, et al. In vivo imaging of murine retinal ganglion cells. J Neurosci Methods 2008;168:475-478. [PubMed: 18079000]

29. Seeliger MW, Beck SC, Pereyra-Muñoz N, et al. In vivo confocal imaging of the retina in animal models using scanning laser ophthalmoscopy. Vision Res 2005;45:3512-3519. [PubMed: 16188288]

30. Paques M, Sirnonutti M, Roux MJ, et al. High resolution fundus imaging by confocal scanning laser ophthalmoscopy in the mouse. Vision Res 2006;46:1336-1345. [PubMed: 16289196]

31. Biss DP, Sumorok D, Burns SA, et al. In vivo fluorescent imaging of the mouse retina using adaptive optics. Opt Lett 2007;32:659-661. [PubMed: 17308593]

32. Kawaguchi I, Higashide T, Ohkubo S, Takeda H, Sugiyama K. In vivo imaging and quantitative evaluation of the rat retinal nerve fiber layer using scanning laser ophthalmoscopy. Invest Ophthalmol Vis Sci 2006;47:2911-2916. [PubMed: 16799033]

33. Walsh MK, Quigley HA. In vivo time-lapse fluorescence imaging of individual retinal ganglion cells in mice. J Neurosci Methods 2008;169:214-221. [PubMed: 18199485]

34. Zambarakji HJ, Keegan DJ, Holmes TM, et al. High resolution imaging of fluorescein patterns in RCS rat retinae and their direct correlation with histology. Exp Eye Res 2006;82:164-171. [PubMed: 16054136]

35. Paques M, Guyomard JL, Simonutti M, et al. Panretinal, high-resolution color photography of the mouse fundus. Invest Ophthalmol Vis Sci 2007;48:2769-74. [PubMed: 17525211]

36. Zhang X, De Alwis M, Hart SL, et al. High-titer recombinant adeno-associated virus production from replicating amplicons and herpes vectors deleted for glycoprotein H. Hum Gene Ther 1999;10:252737. [PubMed: 10543617]

37. Imanishi Y, Piston DW, Baehr W, Palczewski K. Noninvasive two-photon imaging reveals retinyl ester storage structures in the eye. J Cell Biol 2004;164:373-383. [PubMed: 14745001]

38. Murata H, Aihara M, Chen Y, Ota T, Numaga J, Araie M. Imaging mouse retinal ganglion cells and their loss in vivo by a fundus camera in the normal and ischemia-reperfusion model. Invest Ophthalmol Vis Sci 2008;49(12):5546-5552. [PubMed: 18689704]

39. Leung CK, Lindsey JD, Crowston JG, Chen L, Chiang S, Weinreb RN. Longitudinal profile of retinal ganglion cell damage after optic nerve crush with blue-light confocal scanning laser ophthalmoscopy. Invest Ophthalmol Vis Sci 2008;49(11):4898-4902. [PubMed: 18441315]

40. Liang J, Williams DR, Miller D. Supernormal vision and high resolution retinal imaging through adaptive optics. J Opt Soc Am A 1997;14:2884-2892. 
41. Roorda A, Romero-Borja F, Donnelly WJ, Queener H, Hebert TJ, Campbell MCW. Adaptive optics scanning laser ophthalmoscopy. Opt Express 2002;10:405-412. [PubMed: 19436374]

42. Gray DC, Merigan W, Wolfing JI, et al. In vivo fluorescence imaging of primate retinal ganglion cells and retinal pigment epithelial cells. Opt Express 2006;14:7144-7158. [PubMed: 19529085]

43. Gray DC, Wolfe R, Gee BP, et al. In Vivo Imaging of the Fine Structure of Rhodamine-Labeled Macaque Retinal Ganglion Cells. Invest Ophthalmol Vis Sci 2008;49:467-473. [PubMed: 18172127]

44. Morgan JIW, Dubra A, Wolfe R, Merigan WH, Williams DR. In vivo autofluorescence imaging of the human and macaque retinal pigment epithelial cell mosaic. Invest Ophthalmol Vis Sci 2009;50:1350-1359. [PubMed: 18952914]

45. Zolotukhin S, Byrne BJ, Mason E, et al. Recombinant adeno-associated virus purification using novel methods improves infectious titer and yield. Gene Ther 1999;6:973-985. [PubMed: 10455399]

46. Greenberg KP, Geller SF, Schaffer DV, Flannery JG. Targeted transgene expression in Müller glia of normal and diseased retinas using lentiviral vectors. Invest Ophthalmol Vis Sci 2007;48:18441852. [PubMed: 17389520]

47. DiLoreto D, Grover DA, Del Cerro C, Del Cerro M. A new procedure for fundus photography and fluorescein angiography in small laboratory animal eyes. Curr Eye Res 1994;13:157-161. [PubMed: 8194363]

48. ANSI. American National Standard for the Safe Use of Lasers ANSI Z136.1-2007. Laser Institute of America; 2007.

49. Delori FC, Webb RH, Sliney DH. Maximum permissible exposures for ocular safety (ANSI 2000), with emphasis on ophthalmic devices. J Opt Soc Am A 2007;24:1250-1265.

50. Huxlin KR, Goodchild AK. Retinal ganglion cells in the albino rat: Revised morphological classification. J Comp Neurol 1997;385:309-323. [PubMed: 9268130]

51. Lee, HC. Introduction to color imaging science. Cambridge: Cambridge University Press; 2005. p. 294

52. Chaudhuri A, Hallet PE, Parker JA. Aspheric curvatures, refractive indices and chromatic aberration for the rat eye. Vision Res 1983;23:1351-1361. [PubMed: 6666037]

53. Shroff SA, Fienup JR, Williams DR. Phase-shift estimation in sinusoidally illuminated images for lateral superresolution. J Opt Soc Am A 2009;26:413-424.

54. Chan F, Bradley A, Wensel TG, Wilson JH. Knock-in human rhodopsin-GFP fusions as mouse models for human disease and targets for gene therapy. Proc Natl Acad Sci U S A 2004;101(24):9109-14. [PubMed: 15184660]

55. Dhingra A, Sulaiman $\mathrm{P}, \mathrm{Xu} \mathrm{Y}$, et al. Probing neurochemical structure and function of retinal ON bipolar cells with a transgenic mouse. J Comp Neurol 2008;510(5):484-96. [PubMed: 18671302]

56. Rolling F. Recombinant AAV-mediated gene transfer to the retina: gene therapy perspectives. Gene Ther 2004;11:S26-S32. [PubMed: 15454954]

57. Greenberg KP, Lee ES, Schaffer DV, Flannery JG. Gene delivery to the retina using lentiviral vectors. Adv Exp Med Biol 2006;572:255-66. [PubMed: 17249582]

58. Flannery JG, Zolotukhin S, Vaquero MI, et al. Efficient photoreceptor-targeted gene expression in vivo by recombinant adeno-associated virus. Proc Natl Acad Sci USA 1997;94:6916-6921.

[PubMed: 9192666] 


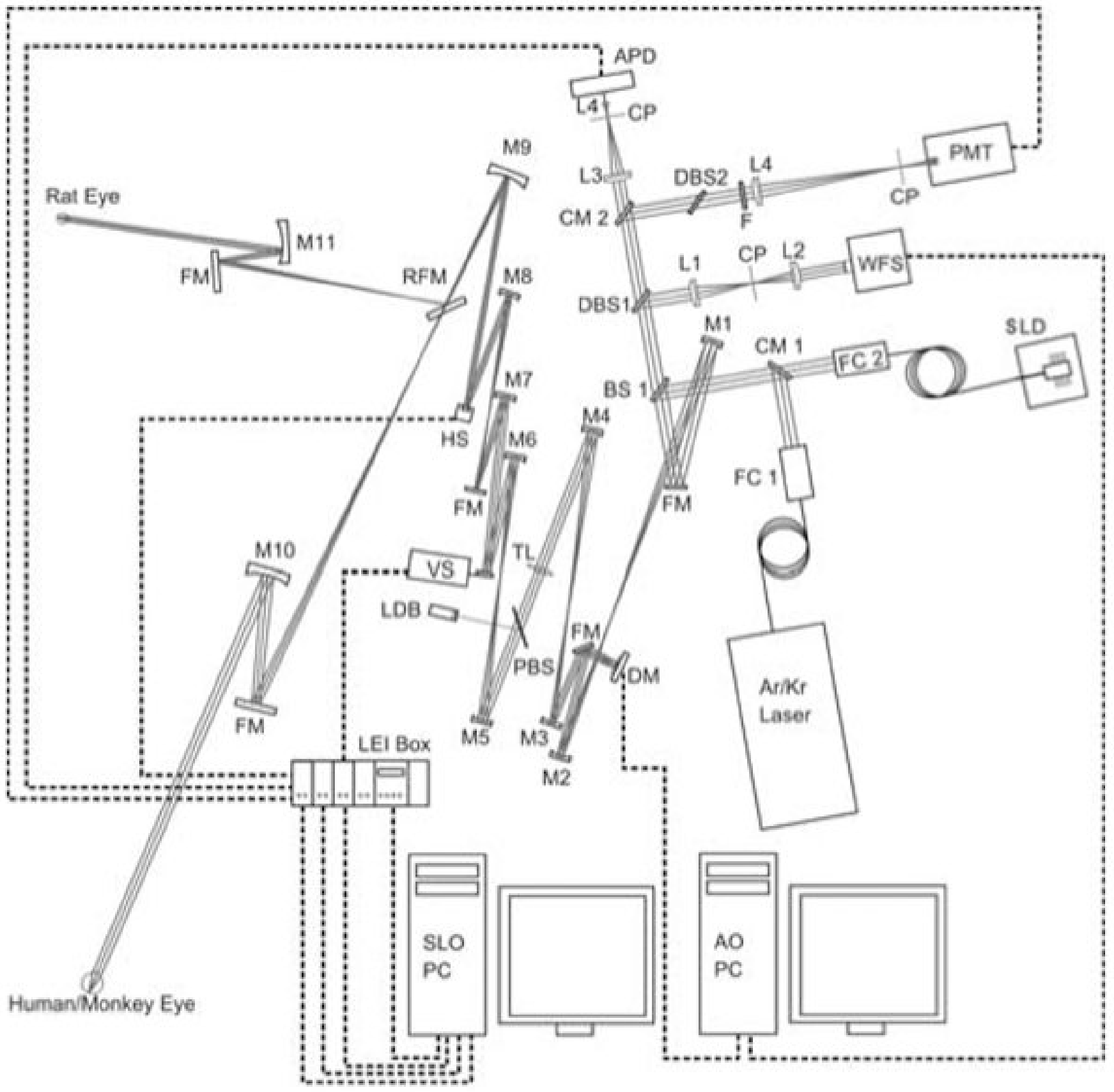

Fig. 1.

The fluorescence adaptive optics scanning laser ophthalmoscope for imaging the rat eye, based on the system previously described by Gray et al. ${ }^{42} \mathrm{~L}$ : lenses, M: spherical mirrors, FM: fold mirrors, CM: cold mirrors, FC: fiber collimators, WFS: wavefront sensor, DBS: dichroic beam splitters, CP: confocal pinholes, SLD: fiber coupled 794-nm superluminescent diode, LDB: laser diode beacon, BS 1: 90/10 beam splitter, DM: MEMS deformable mirror, TL: trial lens plane, PBS: pellicle beam splitter, VS: vertical scanner $(27 \mathrm{~Hz})$, HS: horizontal scanner (15.1 $\mathrm{kHz}$ ), F: band pass fluorescence filter, PMT: photomultiplier tube, APD: avalanche photodiode, LEI Box: scanner control electronics. 

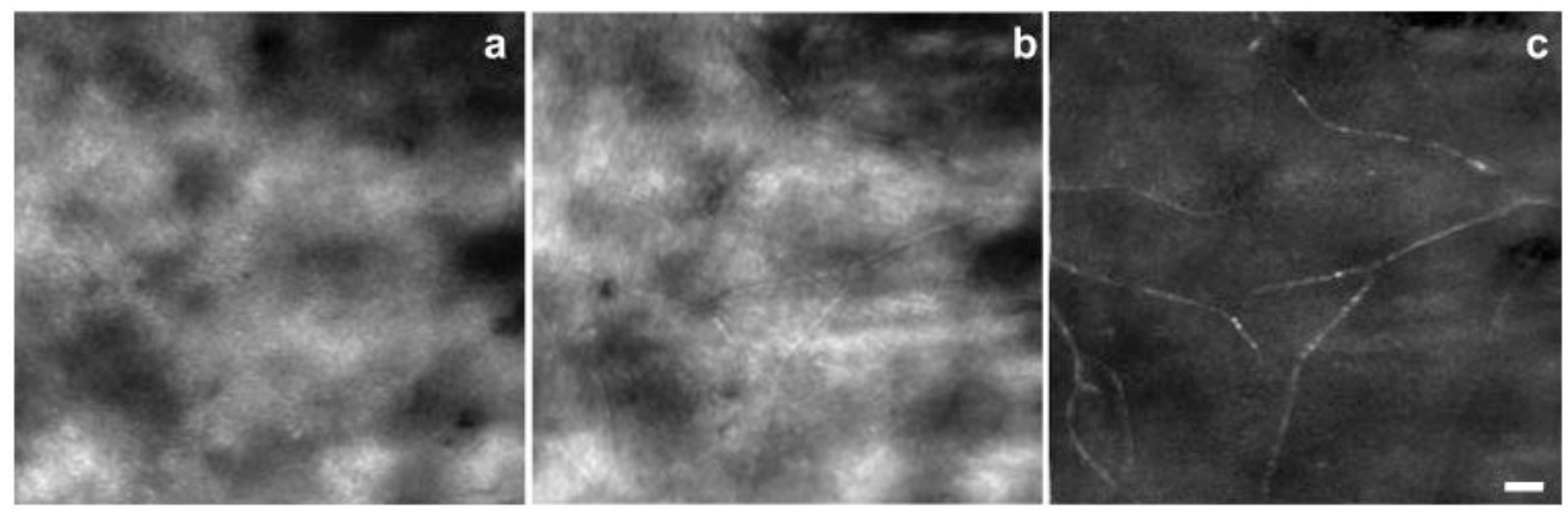

Fig. 2.

In vivo reflectance images of the rat retina taken (a) before and (b) after $\mathrm{AO}$ correction. Both images are the registered sum of 200 frames. (c) Standard deviation image calculated from the registered video acquired after $\mathrm{AO}$ correction. Confocal pinhole diameter was 6.2 Airy disks. Scale bar: $20 \mu \mathrm{m}$. 


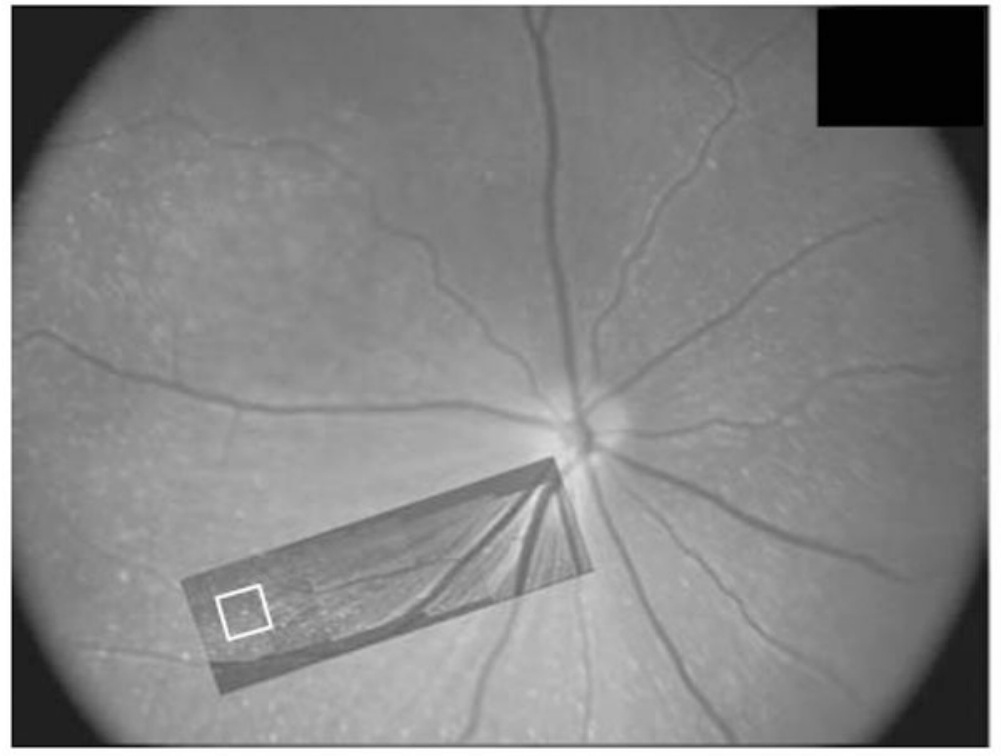

Fig. 3.

In vivo fluorescence fundus photo taken in the rat eye to verify eGFP expression prior to fAOSLO imaging. The rectangular image overlaid on the photo is a montage of low resolution images acquired with the fAOSLO. The fAOSLO montage image was comprised of 5 low resolution images collected over a 9-degree field-of-view; each image was a registered sum of 1000 frames. Both the fundus photos and low resolution fAOSLO montages served as maps to guide imaging over small fields with the high resolution fAOSLO. A magnified, high resolution image of the region denoted by the white square is illustrated in Fig. 5. 

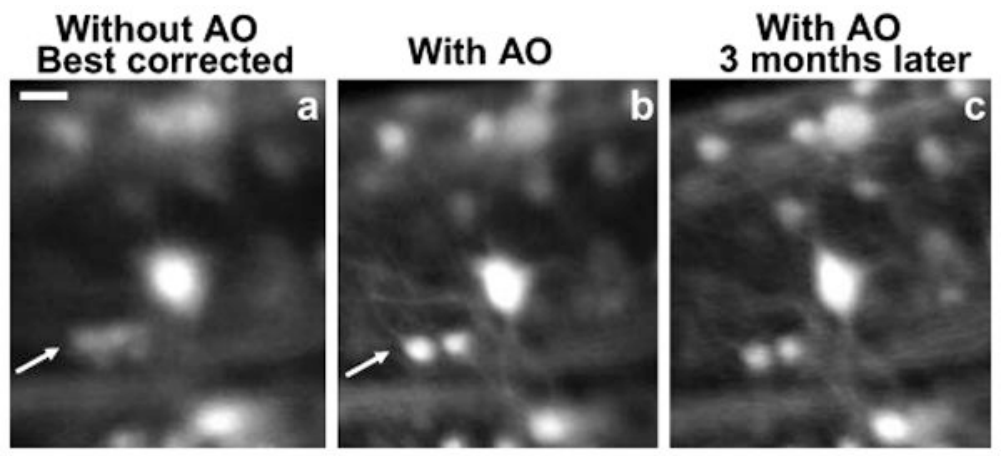

Fig. 4.

In vivo fluorescence images (exciation at $488 \mathrm{~nm}$, peak emission at $507 \mathrm{~nm}$ ) of rat retinal ganglion cells, dendrites and axons labeled with eGFP. (a) Image taken prior to AO correction with a best correction of defocus and astigmatism. Some (but not all) cell bodies can be resolved whereas subcellular features are blurred. (b) Image with AO correction. All cell bodies can be differentiated and subcellular processes are evident. (c) In vivo image of the same retinal location shown in (b) taken 3 months later. The same cellular and subcellular features can be resolved. AO correction was performed over a 1.7- $\mathrm{mm}$ pupil. All images are the registered sum of 1000 frames. All images were contrast enhanced identically for display purposes. Scale bar: $20 \mu \mathrm{m}$. 


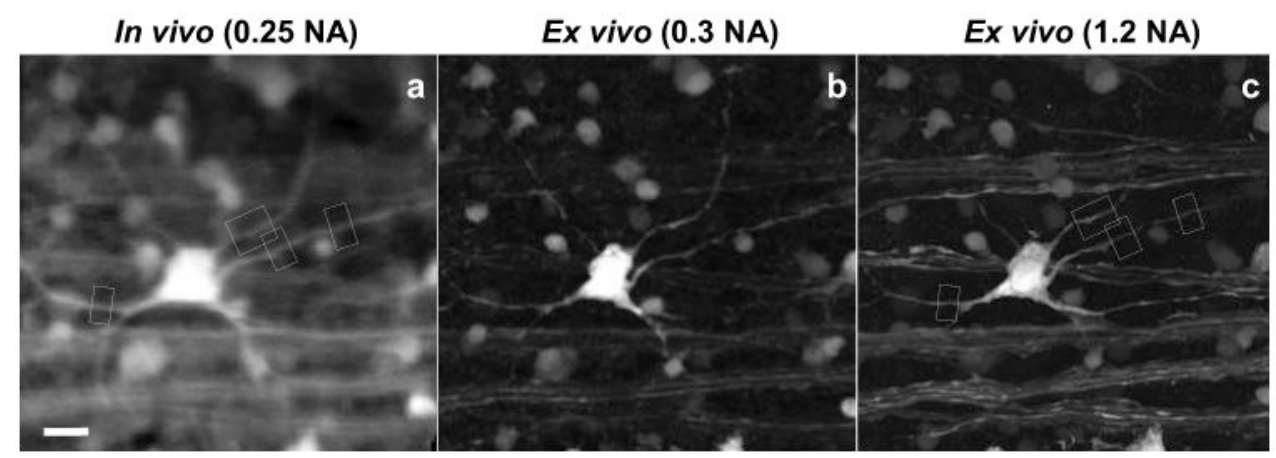

Fig. 5.

Direct comparison of in vivo and ex vivo rat retinal images. (a) In vivo image in a rat retina taken with AO correction over a 0.25 NA. (b) Ex vivo histological image acquired using a confocal microscope with a 0.3 NA objective. (c) Ex vivo histological image acquired using a confocal microscope with a 1.2 NA objective where details of the cell soma, dendrite and axon structures can be seen. The in vivo image is a maximum intensity projection image generated from 3 separate in vivo images taken at different depths, each of which is a registered sum of 1000 frames. Both ex vivo images are maximum intensity projection images generated from a focus stack of 63 images of the ganglion cell layer. Gray rectangles in (a) and (c) indicate locations in which we characterized the in vivo resolution. All images have been rescaled and contrast enhanced for display purposes. Scale bar: $20 \mu \mathrm{m}$. 

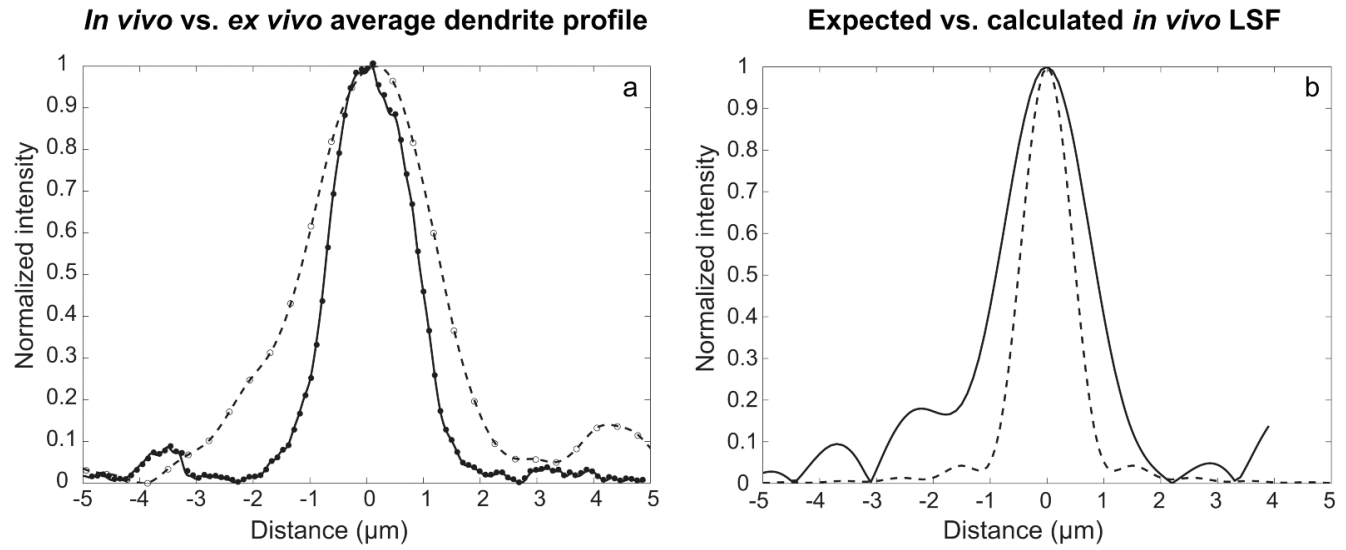

Fig. 6 .

Characterization of in vivo transverse resolution. (a) Average normalized cross-sections of a dendrite imaged in vivo with a 0.25 NA (open circles, dashed line) and ex vivo with a 1.2 NA objective (filled circles, solid line). (b) Calculated in vivo line spread function (LSF) compared with expected theoretical LSF for a 0.25 NA. Solid line: calculated in vivo LSF for a 0.25 NA with a FWHM of $1.77 \mu \mathrm{m}$. For comparison, the expected theoretical LSF for a $0.25 \mathrm{NA}$ eye is plotted (dashed line) with a FWHM of $1.01 \mu \mathrm{m}$. 


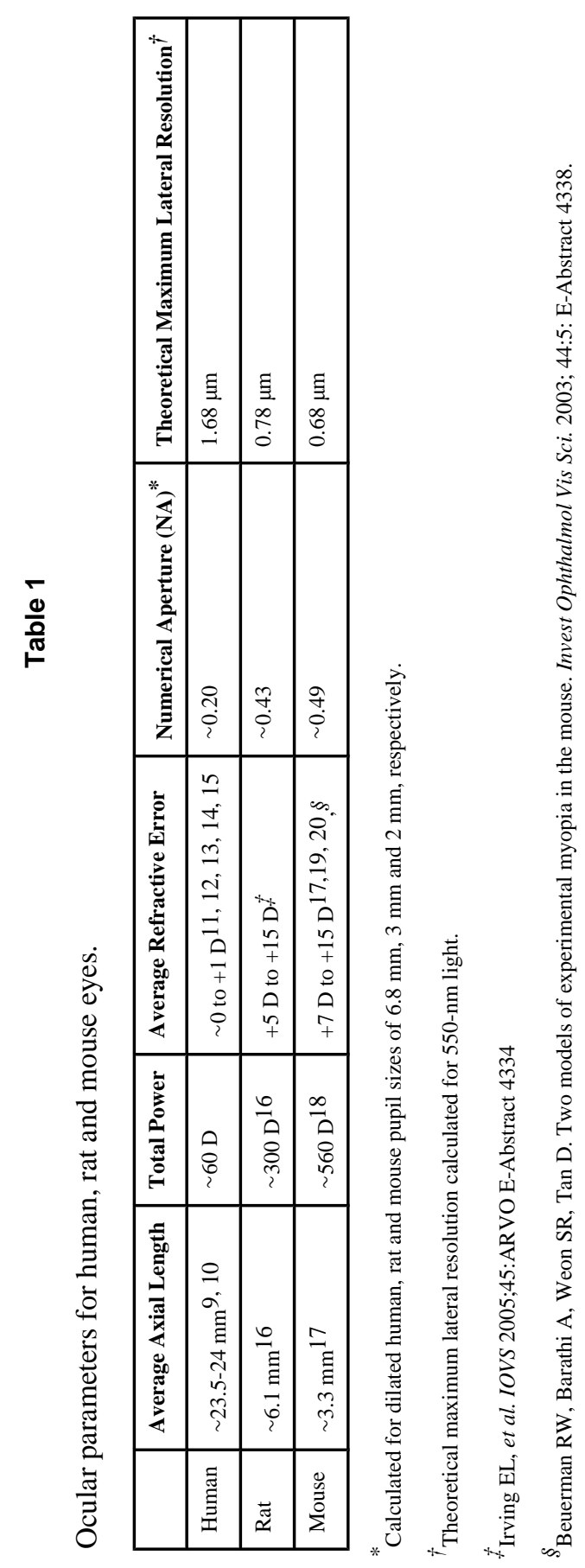

Invest Ophthalmol Vis Sci. Author manuscript; available in PMC 2010 December 1. 